LA GRANA:

REVISTA DE

CIENCIAS DE LA VIDA

pISSN:1390-3799; eISSN:1390-8596

http:/ / doi.org/10.17163/lgr.n30.2019.09
Artículo científico / Scientific paper

DESARROLLO SOSTENIBLE

\title{
DiNÁMICA DE LOS PEQUEÑOS PRODUCTORES DE LECHE EN LA SIERRA CENTRO DE ECUADOR
}

\author{
SMALL-FARMERS DYNAMICS IN THE SIERRA OF ECUADOR \\ Christian Franco-Crespo*i), Lilian Victoria Morales Carrasco ${ }^{*}$, Nelson \\ Rodrigo Lascano Aimacaña $\mathbb{C}^{\circ}$ y Giovanna Alejandra Cuesta Chávez
}

Departamento de Investigación y Desarrollo-DIDE, Universidad Técnica de Ambato, Campus Huachi, Ambato, Ecuador

*Autor para correspondencia: cd.franco@uta.edu.ec

Manuscrito recibido el 30 de enero de 2019. Aceptado, tras revisión, el 14 de agosto de 2019. Publicado el 1 de septiembre de 2019.

\begin{abstract}
Resumen
La estructura agraria en el Ecuador tiene una marcada diferenciación debido a la tenencia y acceso a la tierra. El crecimiento de la producción pecuaria dentro de las pequeñas y medianas explotaciones ha sido constante en los últimos años, así como la feminización del campo. En la serranía del Ecuador, estos procesos pueden observarse con mayor detalle, especialmente en aquellas provincias que se encuentran en el centro del país. Esto ha motivado a analizar la dinámica de los pequeños productores de las provincias de Chimborazo, Cotopaxi y Tungurahua, por medio de la aplicación de una encuesta a 793 agricultores, donde se revisan las características socio-productivas relacionadas con la producción de leche de ganado bovino. Los resultados demuestran que, comparativamente, la producción de leche se concentra en extensiones entre 0,5 y 1 hectárea, siendo la provincia de Tungurahua en donde existe una mayor distribución del tamaño de las explotaciones; sin embargo, la provincia de Chimborazo mantiene un rango de ingresos familiares superiores a las otras dos provincias. Se concluye que los incentivos sobre factores que mejoren la modernización de las explotaciones pueden incidir directamente en los ingresos familiares que no superan el $40 \%$ la remuneración básica mensual del Ecuador.
\end{abstract}

Palabras clave: Producción lechera, economía familiar, Tungurahua, caracterización agricultura familiar.

\begin{abstract}
The agrarian structure in Ecuador has an unequal access to land. The growth of livestock production within small and medium farms has been constant in recent years, as well as the feminization of the agriculture. In the Sierra of Ecuador, these processes can be observed with more detail, especially in provinces located in the Sierra center. This has motivated to analyze the dynamics of the small producers of the provinces of Chimborazo, Cotopaxi and
\end{abstract}


Tungurahua. This work shows the results of a survey that allows to analyze, from a sample of 793 farmers, the socioproductive characteristics of the bovine milk farms. Results show that, comparatively, milk production is concentrated in extensions between 0.5 and 1 hectares, being the province of Tungurahua the one with more size distribution of the farms; however, the province of Chimborazo maintains a higher range of family income than the other two provinces. Finally, it is concluded that incentives on factors that improve the modernization of farms can affect family income that does not exceed $40 \%$ of the basic monthly remuneration.

Keywords: Milk production, family income, Tungurahua, household-farming characterization

Forma sugerida de citar: Franco-Crespo, C., Morales C., L.V., Lascano A., N.R. y Cuesta Ch., G.A. (2019). Dinámica de los pequeños productores de leche en la Sierra centro de Ecuador. La Granja: Revista de Ciencias de la Vida. Vol. 30(2):103-120. http:/ / doi.org/10.17163/lgr.n30. 2019.09.

IDs Orcid:

Christian Franco-Crespo: http:/ / orcid.org/0000-0002-4818-4350

Lilian Victoria Morales Carrasco: http:/ / orcid.org/0000-0001-7026-4544

Nelson Rodrigo Lascano Aimacaña: https:/ / orcid.org/0000-0001-6735-8873

Giovanna Alejandra Cuesta Chávez: http://Orcid.org/0000-0002-0626-9196 


\section{Introducción}

La agricultura es un importante rubro dentro de las economías de los países. La producción pecuaria, en muchos casos, resulta más importante que la agricultura, ya que provee de leche y carne con proteínas y enzimas de alta demanda en la población. Según Steinfeld y Chilonda, (2006), desde los años 90 se ha incrementado el consumo de carne y leche de bovino, a medida que los ingresos de la clase media de países en desarrollo aumentan. Los países productores como China, India y Brasil representan el $65 \%$ de producción de carne y el $52 \%$ de producción de leche a nivel mundial. Se estima que para el año 2024 los países en desarrollo ampliarán su oferta cerca del $80 \%$ del mercado mundial (OECD-FAO, 2015). En cuanto a Latinoamérica, Brasil y Argentina se encuentran a la cabeza de la oferta pecuaria, mientras que Colombia posee el 58\% (5,8 millones de vacas) de la producción pecuaria (Ormel, 2013). Por su parte, Ecuador, registró en el año 2016 alrededor de 4,1 millones de cabezas de ganado bovino por encima de países como Perú o Bolivia (Corporación Financiera Nacional, 2017).

La producción de leche en Ecuador se concentra en la provincia de Pichincha con más de 845000 litros/año (2016), en segundo lugar, está la provincia de Azuay con 561000 litros/año y, en tercer lugar, Cotopaxi con cerca de 484000 litros/año. La Sierra andina de Ecuador abarca la mayor cantidad de producción de leche a nivel nacional, lo que equivale al $64 \%$ de la producción, mientras que la región Costa ocupa el 30\%, y la región Oriental mantiene un 6\% (INEC, 2016). Según el Instituto Nacional de Estadísticas y Censos (INEC, 2016), el $73 \%$ de la leche que se produce (3,86 millones de litros) se destina a la comercialización. En este periodo, las ventas del sector lácteo sumaron USD 23 millones, sin considerar los ingresos por exportaciones.

La producción lechera mantiene una estructura definida en cada región en Ecuador. Por ejemplo, en la Sierra, las unidades productivas tienen un tamaño de tres hectáreas en promedio (Requelme y Bonifaz, 2012). Sin embargo, se identifican tres niveles de producción entre 1-5 ha, 7-20, y entre 20-120 hectáreas. Además, Requelme y Bonifaz, (2012) resaltan que el promedio de producción de leche en el primer nivel (1-5 ha) es de 4,7 litros/vaca/día. Por este motivo, el presente trabajo tiene como pro- pósito analizar las dinámicas de los pequeños productores de leche de tres provincias que componen la Sierra centro de Ecuador: Cotopaxi, Tungurahua y Chimborazo, mediante un estudio que comprende 793 encuestas realizadas a pequeños productores con la finalidad de conocer las dinámicas socioproductivas, partiendo de la necesidad de profundizar los estudios que analicen a las pequeñas explotaciones de leche, ingresos y distribución del hato en la Sierra centro.

\subsection{Revisión de la literatura}

La agricultura es uno de los sectores que genera empleo, en especial en países en desarrollo. En muchos casos, esto sucede debido a la falta de tecnificación de las unidades productivas, lo que requiere mayor mano de obra, tanto de tipo asalariada como familiar (Sumpsi, 2010). Por lo que, la agricultura es una fuente de ingresos, si no es la única, de las familias campesinas. En América Latina se identifican dos sistemas de producción: la agricultura extensiva de tipo agroexportadora, y la agricultura intensiva o agricultura familiar. Esta división está marcada por el desigual acceso a factores de producción como: tierra, riego, crédito o información, limitando la capacidad de los agricultores, así como su desarrollo económico (Franco-Crespo y Sumpsi Viñas, 2017). A la agricultura familiar se le atribuye el $65 \%$ de los alimentos que llegan a los mercados locales. Sin embargo, la reticencia al riesgo ha permitido la adaptación de los sistemas de producción para obtener ingresos familiares a corto plazo por medio de la diversificación de la producción familiar. Por una parte, la producción de hortalizas y vegetales se ha convertido en una fuente de ingresos en ciclo cortos de producción. Inclusive, se han establecido explotaciones familiares de producción de leche a cargo de las mujeres que genera ingresos semanales (Glikman, 1991).

La FAO, (2013) concluye que los procesos económicos a nivel territorial han provocado una alta concentración de la feminización de la agricultura. En este sentido, el rol de la mujer dentro de las actividades agrícolas cada vez es más protagónico dada la necesidad de que los hombres realizan actividades asalariadas en la ciudad. En especial, los pequeños productores han encontrado en la producción de leche una alternativa para obtener ingresos en efectivo de forma semanal, así como es- 
tablecer un sistema de aseguramiento y resiliencia al riesgo que permita afrontar emergencias económicas del núcleo familiar a través de la tenencia de ganado (Mena-Vásconez, Boelens y Vos, 2016).

Entonces, la agricultura familiar tiene un espacio importante dentro de la estructura agrícola de Ecuador y de otros países de Sudamérica. La subdivisión de la tierra, así como las limitaciones generadas por las fallas del mercado a las que se enfrenta este grupo de agricultores, ocasiona que se enmarque a la agricultura familiar en un ambiente de desigualdad en el acceso a los recursos. Como tal, la agricultura familiar se concentra en extensiones de entre 1 y 5 hectáreas (ha), con un aporte mayoritario de uso de mano de obra de tipo familiar para las tareas agrícolas (Schejtman, 2008).

En el caso de Ecuador, se registran para el año 2013, alrededor de 377434 unidades productivas que poseen entre 1 y 20 hectáreas. Es decir, el $89 \%$ de las unidades productivas, categorizadas como pequeños agricultores, poseen alrededor del 55\% del total de superficie agrícola disponible (MAGAP, 2016). Si se detalla al grupo de pequeños agricultores que poseen entre 1 y 5 hectáreas, se observa que en este rango se concentra el $69 \%$ de productores con apenas el 5,7\% de tenencia de tierra (MAGAP, 2016)(Tabla 1).

En la Tabla 1, se puede observar una comparación de la producción de leche entre las provincias de Cotopaxi, Tungurahua y Chimborazo. La comparación entre los años 2015 y 2017 demuestra una inestabilidad en la producción en las tres provincias. Además, en cuanto al rendimiento que se mide en el número de litros (l) y la Unidad Bovina Adulta (UBA), se observa que la provincia de Tungurahua posee un mayor rendimiento $(8,711 / \mathrm{UBA})$, Cotopaxi en segundo lugar $(8,051 / \mathrm{UBA})$ y Chimborazo con el menor rendimiento $(6,651 / \mathrm{UBA})$.

El acceso limitado a la tierra es la principal característica de la pequeña agricultura, la misma que se concentra en la Sierra centro de Ecuador. Así también, el empleo de mano de obra y la presencia de fallas de mercado influyen en el comportamiento de los agricultores (Wiggins, Kirsten y Llambí, 2010; Mumba y col., 2011; Louhichi y Paloma, 2014), en especial sus dinámicas productivas y económicas en torno a la producción de leche en pequeños productores (De Janvry y Sadoulet, 2006; Grisa y Schneider, 2008; Murphy y col., 2014).

Tabla 1. Descripción de las características de la producción lechera en la zona Sierra centro del Ecuador entre los años 2015 y 2017.

\begin{tabular}{|c|c|c|c|c|c|c|c|}
\hline \multirow{3}{*}{ Año } & \multirow{3}{*}{ Descripción } & \multicolumn{6}{|c|}{ Provincias } \\
\hline & & \multicolumn{2}{|c|}{ Cotopaxi } & \multicolumn{2}{|c|}{ Tungurahua } & \multicolumn{2}{|c|}{ Chimborazo } \\
\hline & & Cantidad & Variación( \%) & Cantidad & Variación( \%) & Cantidad & Variación( \%) \\
\hline 2016 & $\begin{array}{l}\text { Posicionamiento de } \\
\text { la Producción } \\
\text { Nacional de leche* }\end{array}$ & $7.00 \%$ & & $6.00 \%$ & & $7.00 \%$ & \\
\hline 2015 & Producción de & 529614 & & 330239 & & 405036 & \\
\hline 2016 & leche cruda & 483699 & -9 & 355679 & 8 & 458181 & 13 \\
\hline 2017 & (litros) & 514759 & 6 & 297060 & -16 & 431325 & -6 \\
\hline 2015 & Número de & 234615 & & 120199 & & 208509 & \\
\hline 1016 & cabezas de & 221960 & -5 & 126754 & 5 & 228500 & 10 \\
\hline 2017 & ganado & 254709 & 15 & 108133 & -15 & 222316 & -3 \\
\hline 2015 & Número de & 65673 & & 39505 & & 59990 & \\
\hline 2016 & vacas & 61179 & -7 & 40470 & 2 & 72524 & 21 \\
\hline 2017 & ordeñadas & 63932 & 4 & 34103 & -16 & 64846 & -11 \\
\hline
\end{tabular}

* Índice a nivel nacional

Fuente: (INEC, 2016; MAGAP, 2016) 
Por otra parte, los estudios relacionados con la descripción de las características de los pequeños productores de leche se enfocan en un análisis de variables a nivel socioeconómico que considera al cantón como la estructura en donde se producen las dinámicas de intercambio entre los productores rurales y las urbes (Escobar y Berdegué, 1990; Tobar, 2010). Es así que Landín, en Escobar y Berdegué, (1990), propone la tipificación de los productores lecheros en el Ecuador a partir de estudios anteriores (Barsky, 1984; ILDES, 1988). En las explotaciones entre 2300 y $2800 \mathrm{msnm}$, existe una mayor presencia de explotaciones medianas ( $>10 \mathrm{ha}$ ) y grandes (>50) (Larrea y col., 2008), mientras que entre 2900 y $3500 \mathrm{msnm}$, en donde existe déficit de riego, se ubican las explotaciones con menos de 5 ha(Bretón, 2012).
En el Ecuador se identifica un grupo de productores rentistas, los cuales no participan en procesos de mejora e innovación tecnológica, y, por otra parte, existen otros grupos de productores en donde el nivel de ingresos está en proporción directa con la capacidad de inversión tecnológica (Barragán, 2010). Entonces, en las provincias de Cotopaxi, Tungurahua y Chimborazo resalta el rol de la agricultura y en especial de la producción de leche dentro de las unidades familiares de producción agrícola (Chiriboga, 2003). Por su estructura, estas provincias poseen características de participación de agricultura familiar, además de las explotaciones que cuentan con mano de obra asalariada. Esto determina que la agricultura se convierte en una fuente de ingresos familiares (Louhichi y Paloma, 2014).

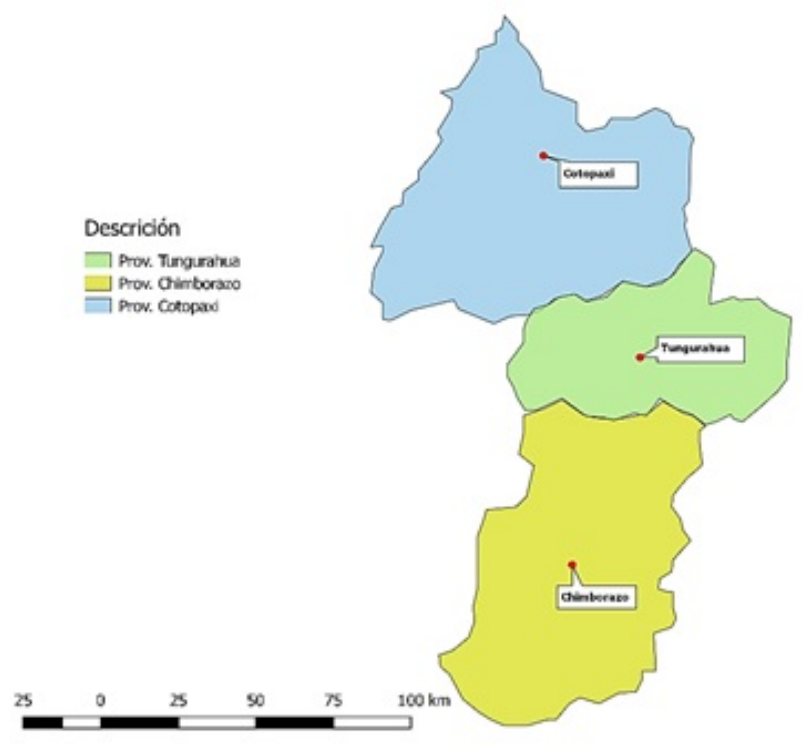

Figura 1. Identificación de las provincias participantes del estudio entre junio y octubre del 2018.

\section{Materiales y Métodos}

El presente estudio se basa en un trabajo de investigación coordinada en tres provincias de la Sierra centro del Ecuador: Cotopaxi, Tungurahua y Chimborazo. El propósito de esta investigación es reconocer las características de las pequeñas explotaciones de producción de leche de manera comparativa entre provincias. Para cumplir con este objetivo, se estableció una muestra en base a la información del Ministerio de Agricultura, Ganadería y Pesca (MA-
GAP) respecto de las bases de datos de productores de leche cruda de las tres provincias al año 2017. También se analizaron las condiciones de tipificación según Landín, en Escobar y Berdegué, (1990).

El instrumento de levantamiento de información fue aplicado según la muestra bajo selección al azar. Los componentes de la encuesta se fijaron en la recopilación de información de la situación socioeconómica de los productores, así como su relación con los factores de producción y acceso al mercado. 
La fórmula empleada (Mantilla, 2015) para el cálculo de la muestra se indica en la ecuación 1 . En donde $n=$ a la muestra, $Z=$ es la desviación del valor medio que aceptamos para lograr el nivel de confianza deseado $(95 \%), p=$ proporción de la población, y $e$ $=$ es el margen de error aceptado $(3 \%)$.

$$
n=\frac{Z^{2} p(1-p)}{e^{2}}
$$

La encuesta fue aplicada entre los meses de junio a octubre del año 2018. En total se recopilaron 793 encuestas. En este sentido, se procedió a aplicar la fórmula del muestreo aleatorio simple a una población de 28270 productores de Cotopaxi (Salcedo, Latacunga), Tungurahua (Pelileo, Píllaro), Chimborazo (Riobamba, Chunchi y Guamote). Las encuestas aplicadas por provincia suman 366 en Cotopaxi, 321 en Tungurahua y 106 en Chimborazo.
El formulario de encuesta tuvo como prueba piloto en el año 2015 la aplicación en la parroquia de San Andrés, del cantón Píllaro de la provincia de Tungurahua. La estructura del instrumento tuvo como referencia la Encuesta de Condiciones de Vida, desarrollada por el INEC para el año 2015. La información y evidencias reposan en los archivos del Proyecto 'La cadena productiva de la leche y la productividad de Cotopaxi, cantón Latacunga' y 'Cadena productiva del sector lechero en la provincia de Tungurahua, cantón Píllaro'. Los registros de los productores se codificaron de acuerdo con cada provincia y se realizaron preguntas de comprobación en los casos de producción e ingresos para más certeza de los resultados obtenidos. Para la validación del cuestionario se aplicó una prueba piloto con una muestra total de 10 productores por cantón con un total de 140, posterior a la prueba piloto se realizaron los ajustes al instrumento aplicado. De esta forma, se plantearon 8 bloques de preguntas.

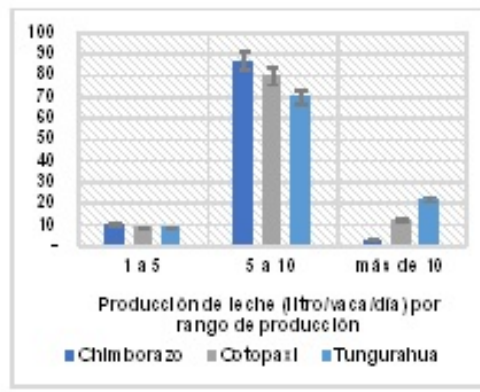

(a)

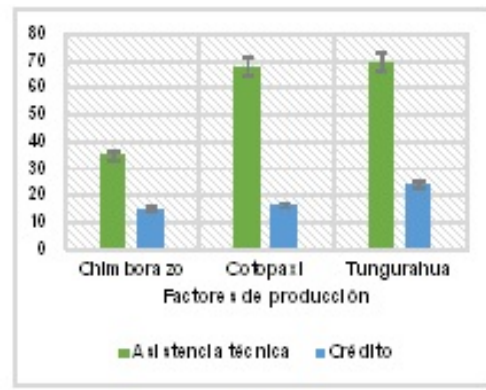

(c)

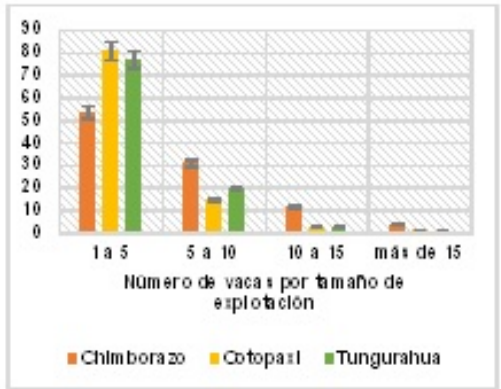

(b)

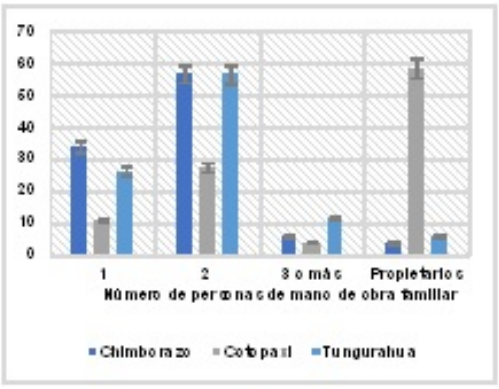

(d)

Figura 2. Resumen de los datos obtenidos de la muestra de las provincias de Chimborazo, Cotopaxi y Tungurahua (a) Producción de leche por rango de producción, (b) Número de vacas por tamaño de explotación, (c) Factores de producción que tienen acceso los productores, y (d) Número de personas de mano de obra familiar. 
Tabla 2. Estadístico descriptivo de la muestra.

\begin{tabular}{|c|c|c|c|c|c|c|c|c|c|c|c|}
\hline Estadísticos & Crédito & $\begin{array}{c}\text { Tipo de } \\
\text { Alimentación } \\
\text { (Balanceados) }\end{array}$ & $\begin{array}{c}\text { Tipo de } \\
\text { Alimentación } \\
\text { (Banano) }\end{array}$ & $\begin{array}{l}\text { Mano de } \\
\text { Obra } \\
\text { Familiar }\end{array}$ & $\begin{array}{c}\text { Mano de } \\
\text { Obra } \\
\text { Contratada }\end{array}$ & $\begin{array}{c}\text { Mano de } \\
\text { Obra Eventual } \\
\text { Contratada }\end{array}$ & $\begin{array}{c}\text { Cantidad } \\
\text { Hectáreas } \\
\text { Cultivadas } \\
\text { de Pasto }\end{array}$ & $\begin{array}{c}\text { Cantidad } \\
\text { Hectáreas } \\
\text { Naturales } \\
\text { de Pasto }\end{array}$ & Regadío & $\begin{array}{l}\text { Fuente } \\
\text { Regadío }\end{array}$ & $\begin{array}{c}\text { Acceso a } \\
\text { Asistencia } \\
\text { Técnica }\end{array}$ \\
\hline Media & 2563 & 1295 & 1842 & 1704 & 1176 & 1333 & 469 & 854 & 1081 & 2553 & 1359 \\
\hline Error típico & 78 & 20 & 16 & 29 & 128 & 142 & 29 & 30 & 10 & 30 & 17 \\
\hline Mediana & 2000 & 1000 & 2000 & 2000 & 1000 & 1000 & 180 & 625 & 1000 & 3000 & 1000 \\
\hline Moda & 2000 & 1000 & 2000 & 2000 & 1000 & 1000 & 90 & 350 & 1000 & 3000 & 1000 \\
\hline $\begin{array}{c}\text { Desviación } \\
\text { estándar }\end{array}$ & 903 & 456 & 365 & 694 & 529 & 492 & 527 & 666 & 273 & 834 & 480 \\
\hline $\begin{array}{l}\text { Varianza de } \\
\text { la muestra }\end{array}$ & 815 & 208 & 134 & 482 & 279 & 242 & 277 & 443 & 74 & 696 & 231 \\
\hline Curtosis & -1032 & -1188 & 1532 & 3593 & 9795 & -1650 & 4874 & 3249 & 7533 & -235 & -1659 \\
\hline $\begin{array}{l}\text { Coeficiente de } \\
\text { asimetría }\end{array}$ & 921 & 904 & -1878 & 1150 & 3136 & 812 & 2066 & 1957 & 3085 & -1329 & 587 \\
\hline Rango & 3000 & 1000 & 1000 & 5000 & 2000 & 1000 & 2960 & 3750 & 1000 & 2000 & 1000 \\
\hline Mínimo & 1000 & 1000 & 1000 & 1000 & 1000 & 1000 & 40 & 100 & 1000 & 1000 & 1000 \\
\hline Máximo & 4000 & 2000 & 2000 & 6000 & 3000 & 2000 & 3000 & 3850 & 2000 & 3000 & 2000 \\
\hline
\end{tabular}

La encuesta se aplicó en las parroquias de las tres provincias. En el cuestionario se recabó la información en función de un grupo de variables e indicadores. Para la validación de correlación del instrumento se analizaron los resultados tabulados mediante la aplicación del Alpha de Cronbach y se observa Cotopaxi $(0,561)$, Chimborazo $(0,686)$ y Tungurahua $(0,657)$. Para el procesamiento estadístico, así como para la obtención de correlaciones, se utilizó Excel estableciendo gráficas comparativas, correlaciones simples y un análisis de medida de variación y valor cuadrático general, respectivamente. Las variables corresponden a los factores de producción, así como de parámetros relacionados con la tecnificación, como pastos y raza de gana-

\section{Resultados y Discusión}

El estudio identifica que el $92,01 \%$ de los productores en la provincia de Cotopaxi de hasta 1 hectárea poseen entre 1 y 10 unidades bovinas en producción. En el caso de la provincia de Tungurahua, el $86,36 \%$ posee entre 1 y 5 unidades bovinas. Así también, en la provincia de Chimborazo, el 82,64\% se encuentra en el mismo rango de unidades bovinas. En general, se identifican las explotaciones por tamaño de tierra, dada en tres categorías: (1) 0,1 a 0,5, ha (2) más de 0,5 a 1 ha, y (3) más de 10 hectáreas. En la Tabla 2 se observan el sistema de producción, recursos disponibles (número de vacas, superficie cultivada, riego y mano de obra) y otros fac- do, relativos con la mejora de la productividad. En los análisis planteados se seleccionan las relaciones que evidencian un nivel de significatividad menor a 0,05 con un nivel de confianza del $95 \%$, lo que permite realizar una inferencia a toda la población. La Tabla 2 presenta los resultados descriptivos de los productores de leche de las tres provincias.

En la Tabla 3 se presenta el estadístico descriptivo de la muestra, considerando las variables estudiadas en este trabajo. En esta tabla se muestran los valores estadísticos que indican la distribución de los datos para el total de datos obtenidos mediante encuestas en las tres provincias.

tores de producción (crédito y asistencia técnica), hallados entre los productores. Por otra parte, en la Tabla 4 se presenta una descripción de los factores socioeconómicos de la población analizada. Aquí se puede ver la composición por mano de obra dentro de la explotación, fuente de crédito y asistencia técnica. De manera general, se observa que la mano de obra familiar tiene mayor presencia en comparación con la mano de obra contratada, así como la mano de obra eventual. En cuanto a la asistencia técnica en Chimborazo, más de los dos tercios declara no haberla recibido, a diferencia de Cotopaxi y Tungurahua. También, las Cooperativas son la principal fuente de financiamiento en las tres provincias. 
Tabla 3. Caracterización de las explotaciones por provincia, según información recopilada en el año 2018.

\begin{tabular}{|c|c|c|c|c|c|c|c|}
\hline \multirow{4}{*}{ Factor } & \multirow{4}{*}{$\begin{array}{c}\text { Descripción } \\
\text { (ha) } \\
\end{array}$} & \multicolumn{6}{|c|}{ Provincia } \\
\hline & & \multicolumn{2}{|c|}{ Chimborazo } & \multicolumn{2}{|c|}{ Cotopaxi } & \multicolumn{2}{|c|}{ Tungurahua } \\
\hline & & Frecuencia & Porcentaje & Frecuencia & Porcentaje & Frecuencia & Porcentaje \\
\hline & & & $(\%)$ & & $(\%)$ & & $(\%)$ \\
\hline \multirow{4}{*}{$\begin{array}{l}\text { Número } \\
\text { de Vacas }\end{array}$} & 1 a 5 & 57 & 53,77 & 296 & 80,87 & 247 & 76,95 \\
\hline & 5 a 10 & 33 & 31,13 & 55 & 15,03 & 63 & 19,63 \\
\hline & más de 10 & 16 & 15,09 & 15 & 4,1 & 11 & 3,42 \\
\hline & Total & 106 & 100 & 366 & 100 & 321 & 100 \\
\hline \multirow{4}{*}{$\begin{array}{c}\text { Producción } \\
\text { (Litro/Vaca/Día) }\end{array}$} & 1 a 5 & 11 & 10,38 & 31 & 8,47 & 27 & 8,41 \\
\hline & 5 a 10 & 92 & 86,79 & 291 & 79,51 & 224 & 69,78 \\
\hline & más de 10 & 3 & 2,83 & 44 & 12,02 & 70 & 21,81 \\
\hline & Total & 106 & 100 & 366 & 100 & 321 & 100 \\
\hline \multirow{5}{*}{$\begin{array}{c}\text { Cantidad de } \\
\text { Hectáreas } \\
\text { cultivadas } \\
\text { de pasto }\end{array}$} & 0,1 a 0,5 & 33 & 31,13 & 201 & 54,92 & 18 & 5,61 \\
\hline & 0,5 a 1 & - & - & 46 & 12,57 & 40 & 12,46 \\
\hline & más de 1 & - & - & 16 & 4,37 & 8 & 2,49 \\
\hline & No tiene & 73 & 68,87 & 103 & 28,14 & 255 & 79,44 \\
\hline & Total & 106 & 100 & 366 & 100 & 321 & 100 \\
\hline \multirow{5}{*}{$\begin{array}{c}\text { Cantidad de } \\
\text { hectáreas } \\
\text { naturales } \\
\text { de pasto }\end{array}$} & 0,1 a 0,5 & 49 & 46,23 & 28 & 7,65 & 45 & 14,02 \\
\hline & 0,5 a 1 & 57 & 53,77 & 73 & 19,95 & 137 & 42,68 \\
\hline & más de 1 & - & - & 8 & 2,19 & 85 & 26,48 \\
\hline & No tiene & - & - & 257 & 70,22 & 54 & 16,82 \\
\hline & Total & 106 & 100 & 366 & 100 & 321 & 100 \\
\hline \multirow{3}{*}{$\begin{array}{l}\text { Cuenta con } \\
\text { agua para } \\
\text { regadío }\end{array}$} & $\mathrm{Si}$ & 106 & 100 & 347 & 94,81 & 276 & 85,98 \\
\hline & No & & - & 19 & 5,19 & 45 & 14,02 \\
\hline & Total & 106 & 100 & 366 & 100 & 321 & 100 \\
\hline \multirow{4}{*}{$\begin{array}{l}\text { Fuente de } \\
\text { regadío }\end{array}$} & Público & 85 & 80,19 & 22 & 6,01 & 61 & 19,00 \\
\hline & Junta de Agua & 21 & 19,81 & 344 & 93,99 & 218 & 67,91 \\
\hline & $\mathrm{N} / \mathrm{C}$ & - & - & - & - & 42 & 13,08 \\
\hline & Total & 106 & 100 & 366 & 100 & 321 & 100 \\
\hline
\end{tabular}

En resumen, en la figura anterior se resalta que la producción de leche mantiene frecuencias absolutas entre los 5 y 10 1/UBA/día, siendo la provincia de Chimborazo la que presenta mayor producción (Figura 2a). En el caso del número de vacas por explotación la frecuencia absoluta se observa en 1 y 5 UBAs, con mayor cantidad para la provincia de Cotopaxi (Figura 2b). Además, se observa que la asistencia técnica se desarrolla con mayor frecuencia en las provincias de Tungurahua y Cotopaxi (Figura 2c). Finalmente, en cuanto a la mano de obra familiar empleada por explotación, Cotopaxi concentra la mayor cantidad de propietarios directos que trabajan en la finca, mientras que en Chimborazo y Tungurahua se promedia en dos personas de la familia que mantienen una actividad permanente en la explotación (Figura 2d).

Por otra parte, respecto a los pastos no cultivados, el porcentaje tiene la misma relación. Es así que en la provincia de Cotopaxi el $87,16 \%$ de los productores poseen entre 1 y 10 unidades bovinas en producción. En la provincia de Chimborazo, el $96,97 \%$ de los productores se encuentra en este mismo rango; sin embargo, en la provincia de Tungurahua solamente el $67,79 \%$ de los encuestados se encuentran en este rango. En cuanto a la variación entre unidades bovinas y el tamaño de explotación, en la Figura 3a se presenta la concentración de unidades bovinas entre las explotaciones que no superan la 1 ha de extensión, en especial entre aquellas que se ubican entre 0,5 y 1 ha. En comparación con la Figura 3b, existe una dispersión de la tenencia de unidades bovinas entre unidades de menor tamaña a las 0,5 ha hasta 1 ha. (Figura 3).

Mientras, en la Figura 3c se observa que la distribución del número de especies bovinas se distribuye entre las explotaciones de 0,5 ha y 3 ha. Sin embargo, el mayor número está entre las 0,5 y 1 
Tabla 4. Identificación de los factores socioeconómicos por provincia, según información recopilada en el año 2018.

\begin{tabular}{|c|c|c|c|c|c|c|c|}
\hline \multirow[t]{2}{*}{ Factor } & \multirow{2}{*}{ Descripción } & \multicolumn{2}{|c|}{ Chimborazo } & \multicolumn{2}{|c|}{ Cotopaxi } & \multicolumn{2}{|c|}{ Tungurahua } \\
\hline & & Frecuencia & Porcentaje( \%) & Frecuencia & Porcentaje( \%) & Frecuencia & Porcentaje( \%) \\
\hline \multirow{5}{*}{$\begin{array}{c}\text { Cantidad de mano } \\
\text { de obra familiar } \\
\text { en la producción } \\
\text { de leche }\end{array}$} & 1 & 36 & 33,96 & 39 & 10,66 & 84 & 26,17 \\
\hline & 2 & 60 & 56,60 & 100 & 27,32 & 181 & 56,39 \\
\hline & 3 o más & 6 & 5,66 & 14 & 3,83 & 37 & 11,53 \\
\hline & Propietarios & 4 & 3,77 & 213 & 58,20 & 19 & 5,92 \\
\hline & Total & 106 & 100 & 366 & 100 & 321 & 100 \\
\hline \multirow{5}{*}{$\begin{array}{c}\text { Cantidad de la mano } \\
\text { de obra contratada } \\
\text { en la producción de } \\
\text { leche }\end{array}$} & 1 & 4 & 3,77 & 2 & 0,55 & 9 & 2,80 \\
\hline & 2 & - & - & - & - & 1 & 0,31 \\
\hline & 3 o más & - & - & - & - & 1 & 0,31 \\
\hline & No contrata & 102 & 96,23 & 364 & 99,45 & 310 & 96,57 \\
\hline & Total & 106 & 100 & 366 & 100 & 321 & 100 \\
\hline \multirow{5}{*}{$\begin{array}{l}\text { Cantidad de mano } \\
\text { de obra eventual } \\
\text { contratada en la } \\
\text { producción de leche }\end{array}$} & 1 & - & - & 3 & 0,82 & 5 & 1,56 \\
\hline & 2 & - & - & 1 & 0,27 & 3 & 0,93 \\
\hline & 3 o más & - & - & - & - & - & - \\
\hline & No contrata & 106 & 100 & 362 & 98,91 & 313 & 97,51 \\
\hline & Total & 106 & 100 & 366 & 100 & 321 & 100 \\
\hline \multirow{3}{*}{$\begin{array}{c}\text { Acceso a asistencia } \\
\text { técnica }\end{array}$} & $\mathrm{Si}$ & 37 & 34,91 & 248,0 & 67,76 & 223 & 69,47 \\
\hline & No & 69 & 65,09 & 118,0 & 32,24 & 98 & 30,53 \\
\hline & Total & 106 & 100 & 366 & 100 & 321 & 100 \\
\hline \multirow{3}{*}{$\begin{array}{c}\text { Fuentes de } \\
\text { financiamiento } \\
\text { de sus actividades }\end{array}$} & Propias & 90 & 84,91 & 306 & 83,61 & 243 & 75,70 \\
\hline & Crédito & 16 & 15,09 & 60 & 16,39 & 78 & 24,30 \\
\hline & Total & 106 & 100 & 366 & 100 & 321 & 100 \\
\hline \multirow{7}{*}{ Fuente de crédito } & Proveedores & - & - & & - & 1 & 1,28 \\
\hline & $\begin{array}{l}\text { Coop. de Ahorro } \\
\text { y Crédito }\end{array}$ & 11 & 68,75 & 47 & 78,33 & 47 & 60,26 \\
\hline & Financieras & - & - & - & - & 3 & 3,85 \\
\hline & Bancos & 1 & 6,25 & 12 & -2 & 25 & 32,05 \\
\hline & Familiar & 4 & 25,00 & - & - & - & - \\
\hline & No requiere & - & - & 1 & 1,67 & 2 & 2,56 \\
\hline & Total & 16 & 100 & 60 & 100 & 78 & 100 \\
\hline
\end{tabular}

ha. Entonces, en el análisis comparativo se puede mencionar que Cotopaxi mantiene explotaciones de producción lechera con extensiones de menos de 0,5 ha. Por otra parte, en la provincia de Chimborazo estas explotaciones de producción de leche se ubican entre las 0,5 y 1 ha. En cambio, las explotaciones de producción de leche en la provincia de Tungurahua presentan un rango más amplio, aunque persiste la concentración entre 0,5 y 1 ha.

Al analizar esta comparación entre las explotaciones de mayor tamaño (superior a 101 casos analizados), aquellas explotaciones que tienen un mayor tamaño tienden a ser más eficientes en Tungurahua, comparadas con aquellas que se ubican en la provincia de Cotopaxi. Si se analizan estos resultados con la descripción presentada en la Figura 2c y Figura 2d, la asistencia técnica es mayor en las provincias de Tungurahua y Cotopaxi. Sin embargo, la mayor cantidad de mano de obra familiar ocurre en la provincia de Chimborazo.
En la Figura 4 se presentan los resultados de los ingresos directos de la producción de leche consolidado para las tres provincias. De forma comparativa, el número de UBAs en los tres rangos considerados (1-5, 6-10 y 11-20) plantea una condición de productividad en función de la cantidad de ingresos que pueden obtener producto de la venta de la leche. Aquellas explotaciones que poseen entre 1 y 5 vacas obtienen ingresos menores a USD 386,00 por mes. Por otra parte, las explotaciones que mantienen hatos entre 6 y 10 vacas promedian ingresos de entre 386 USD/mes y 900 USD/mes. En último lugar, se encuentran las explotaciones con más de 11 unidades bovinas que generan más de 900 USD/mes. Como caso particular, Tungurahua presenta en el rango de 1-5 UBAs ingresos de más de $\$ 900$. Por otra parte, Cotopaxi presenta en el rango 6-10 UBAs el mismo nivel de ingresos, mientras Chimborazo es la provincia con menor número de UBAs en este rango de ingresos. 

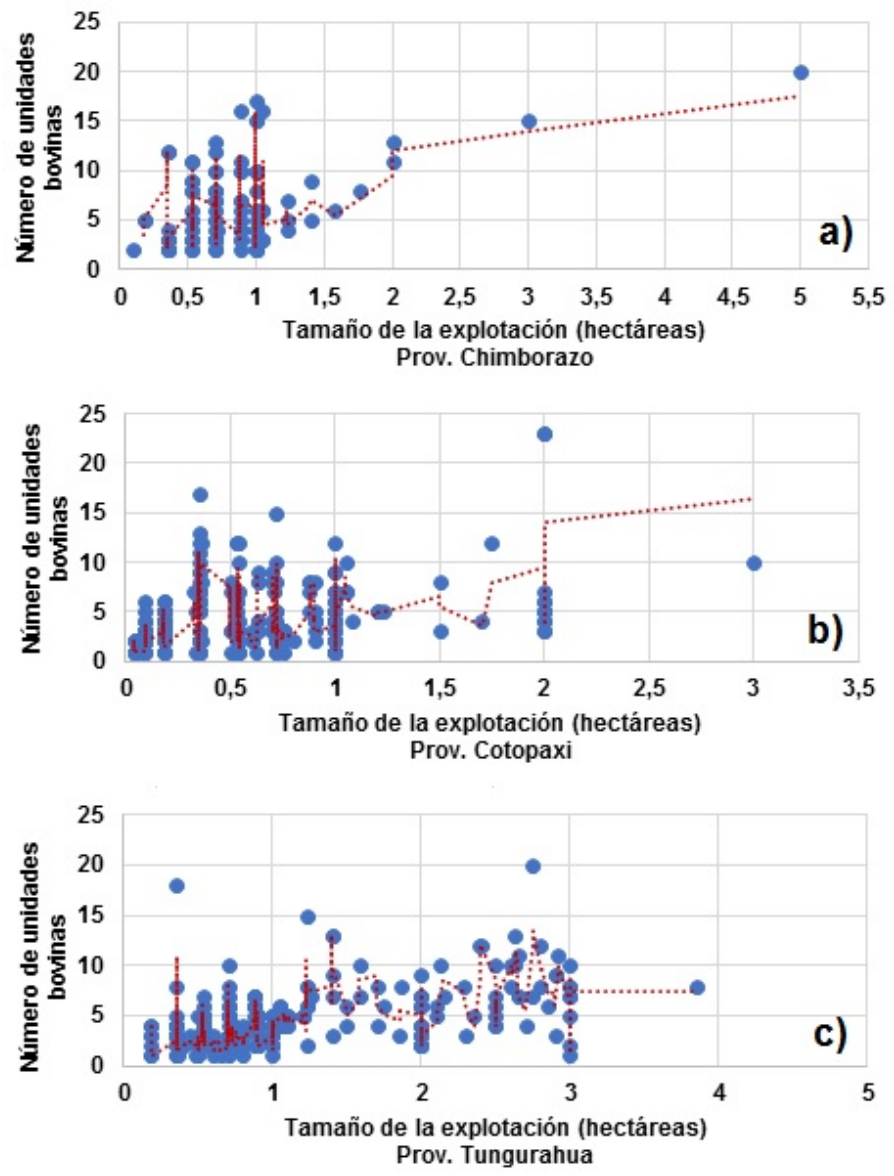

Figura 3. Relación del número de unidades bovinas por tamaño de explotación por cada provincia (a) Unidades bovinas en la provincia de Chimborazo, (b) Unidades bovinas en la prov. de Cotopaxi, y (c) Unidades bovinas en la prov. de Tungurahua.

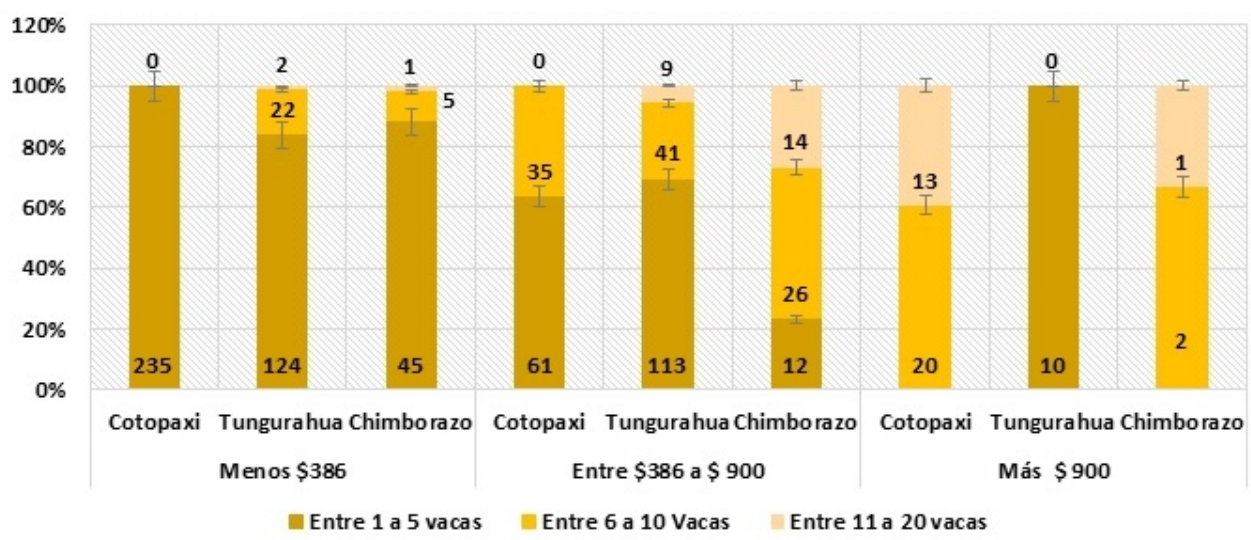

Figura 4. Rango de unidades bovinas y nivel de ingresos por la venta de leche por provincia. 
En relación a los ingresos comparativos que generan las explotaciones de acuerdo con su provincia, aquellas que se encuentran en Chimborazo concentran ingresos de entre $300 \mathrm{USD} /$ mes y 800 $\mathrm{USD} / \mathrm{mes}$, en las explotaciones mayores a 0,5 ha y 1 ha. Las explotaciones de más de 1 ha generan mayores ingresos en esta misma provincia, pero el número de casos es menor Por otra parte, se debe resaltar que las explotaciones de más de 1 ha de Cotopaxi y Tungurahua mantienen una constante en la generación de ingresos (menos unidades bovinas en producción) entre 100 y 200 USD/mes (Figura 5).

Los ingresos familiares que provienen de la venta de leche son más altos en la provincia de Chimborazo con 485 USD/mes. En segundo lugar, la provincia de Tungurahua mantiene explotaciones que permiten el ingreso familiar de más de 360 USD/mes. En cambio, las explotaciones que se ubican en la provincia de Cotopaxi generan un poco más de la mitad de los ingresos que se obtienen en el primer caso, siendo los ingresos por familia de más de 260 USD/mes (Figura 6).
En este caso, las variables que influyen en un mejor rendimiento del hato ganadero son aquellas que han provocado la modernización de las explotaciones. Es decir, se supone que las explotaciones que mantienen estas características de innovación tendrán la capacidad de producir ingresos, incluso si corresponden a extensiones de tamaño pequeño ( 0,5 a 1 ha) o poseen menos unidades bovinas ( 1 a 5 vacas). Los resultados obtenidos permiten evidenciar que la provincia de Chimborazo posee un mayor rendimiento respecto a Cotopaxi y Tungurahua. Si bien los resultados propuestos por el INEC para el año 2015 indican que la provincia de Chimborazo mantiene, de forma comparativa con las otras dos provincias, un menor rendimiento $(6,651 / \mathrm{UBA})$, así también los ingresos analizados que perciben las familias por concepto de la producción y venta de leche (hasta cinco unidades bovinas equivalente al $42,25 \%$ ), alcanzan menos de una remuneración básica unificada (USD394). Al comparar los ingresos familiares por provincia, la provincia de Cotopaxi presenta que las explotaciones analizadas perciben menos de USD260/mes.

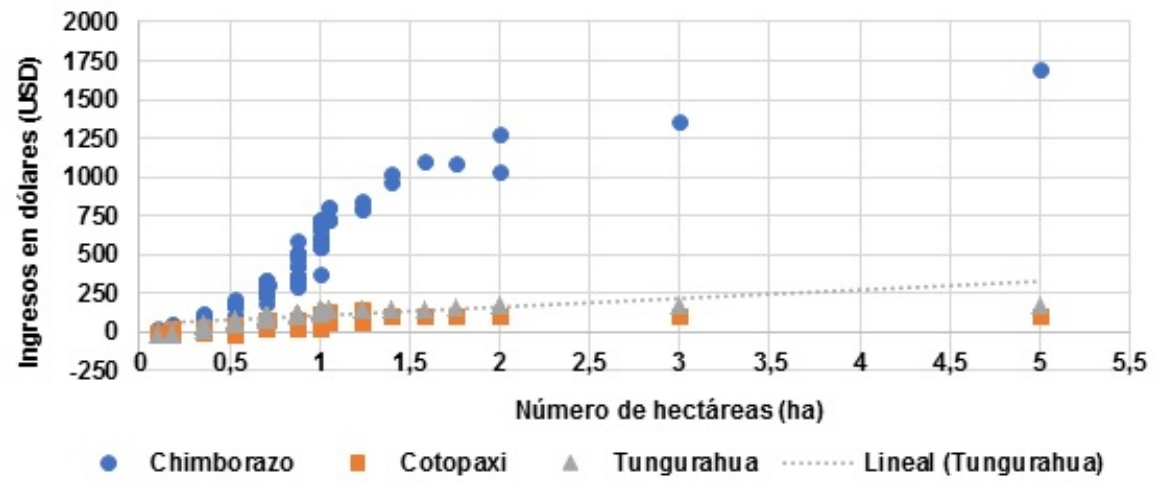

Figura 5. Comparativo de ingresos por provincia.

Por otra parte, en la Tabla 5 se presentan los resultados de la correlación entre variables determinadas al inicio de la aplicación del instrumento de investigación. Aquellas correlaciones significativas corresponden a aquellos factores que tienen relación con la innovación de las explotaciones de pro- ducción pecuaria en las tres provincias. Aquellos factores que se relacionan con superficie de pastos cultivados, mejora genética, alimento complementario, asistencia técnica e instalaciones tecnificadas, aparecen como significativas. 


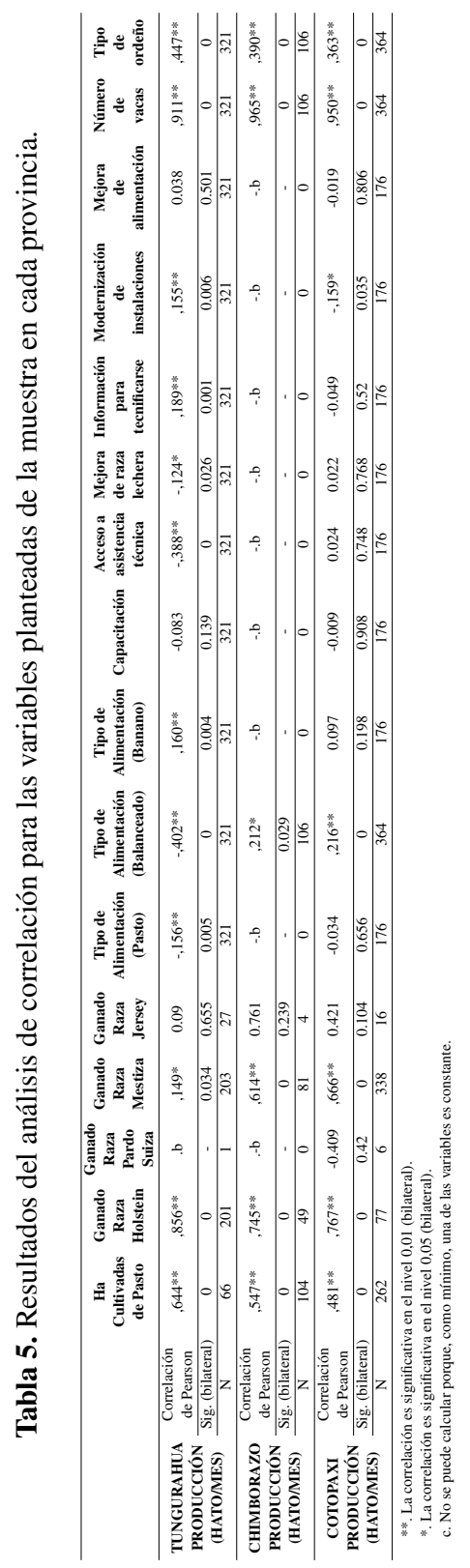


Al aplicar el análisis de medida de variación en conjunto a los datos obtenidos en las tres provincias (Cotopaxi, Tungurahua y Chimborazo), se encuentran diferencias significativas entre ellas, para lo cual se aplica una prueba post-hoc (Tukey), a fin de identificar cuál es la provincia que presenta mayores diferencias respecto de las demás, en función de las variables más significativas (Tabla 6). La Tabla 6, presenta los resultados del análisis de medida de variación entre el grupo de variables identificados con correlación significativa, de tal forma que se toman para realizar post-hoc Tukey que se presentan en las siguientes tablas.

Tabla 6. Resultado de la medida de variación y valor cuadrático para las tres provincias.

\begin{tabular}{|c|c|c|c|c|c|c|}
\hline \multicolumn{2}{|c|}{ Descripción } & Suma de cuadrados & gl & Media cuadrática & $\mathbf{F}$ & Sig. \\
\hline \multirow{3}{*}{$\begin{array}{c}\text { Tipo de Alimentación } \\
\text { del ganado } \\
\text { (Banano) }\end{array}$} & Entre grupos & 10,791 & 1 & 10,791 & 95,985 &, 000 \\
\hline & Dentro de grupos & 55,651 & 495 & 112 & & \\
\hline & Total & 66,443 & 496 & & & \\
\hline \multirow{3}{*}{$\begin{array}{l}\text { Número de vacas } \\
\text { por hato }\end{array}$} & Entre grupos & 528,929 & 2 & 264,465 & 28,875 &, 000 \\
\hline & Dentro de grupos & 7217,336 & 788 & 9,159 & & \\
\hline & Total & 7746,265 & 790 & & & \\
\hline \multirow{3}{*}{$\begin{array}{l}\text { Producción } \\
\text { (Hato/mes) }\end{array}$} & Entre grupos & 40645527,511 & 2 & 20322763,756 & 24,544 &, 000 \\
\hline & Dentro de grupos & 652466338,734 & 788 & 828002,968 & & \\
\hline & Total & 693111866,245 & 790 & & & \\
\hline \multirow{3}{*}{$\begin{array}{c}\text { Cantidad de hectáreas } \\
\text { Cultivadas de } \\
\text { Pasto } \\
\end{array}$} & Entre grupos & 13,419 & 2 & 6,709 & 25,760 & ,000 \\
\hline & Dentro de grupos & 111,738 & 429 & ,260 & & \\
\hline & Total & 125,157 & 431 & & & \\
\hline \multirow{3}{*}{$\begin{array}{c}\text { Cantidad de hectáreas } \\
\text { naturales } \\
\text { de pasto } \\
\end{array}$} & Entre grupos & 23,419 & 2 & 11,709 & 26,975 & ,000 \\
\hline & Dentro de grupos & 175,799 & 405 & 434 & & \\
\hline & Total & 199,217 & 407 & & & \\
\hline \multirow{3}{*}{$\begin{array}{l}\text { Cantidad ganado } \\
\text { raza Holstein }\end{array}$} & Entre grupos & 169,186 & 2 & 84,593 & 9810 & ,000 \\
\hline & Dentro de grupos & 2794,031 & 324 & 8,624 & & \\
\hline & Total & 2963,217 & 326 & & & \\
\hline \multirow{3}{*}{$\begin{array}{c}\text { Cantidad ganado } \\
\text { raza } \\
\text { mestizo/criollo }\end{array}$} & Entre grupos & 571,364 & 2 & 285,682 & 74,019 &, 000 \\
\hline & Dentro de grupos & 2389,073 & 619 & 3,860 & & \\
\hline & Total & 2960,437 & 621 & & & \\
\hline \multirow{3}{*}{$\begin{array}{l}\text { Acceso a } \\
\text { asistencia } \\
\text { técnica }\end{array}$} & Entre grupos & 10,474 & 2 & 5,237 & 24,055 &, 000 \\
\hline & Dentro de grupos & 171,559 & 788 & ,218 & & \\
\hline & Total & 182,033 & 790 & & & \\
\hline \multirow{3}{*}{$\begin{array}{c}\text { Modificación } \\
\text { de instalaciones } \\
\text { del hato }\end{array}$} & Entre grupos & 5,723 & 1 & 5,723 & 26,857 &, 000 \\
\hline & Dentro de grupos & 105,488 & 495 & ,213 & & \\
\hline & Total & 111,211 & 496 & & & \\
\hline
\end{tabular}

En la Tabla 7, se observa que la provincia que presenta mayores diferencias respecto del número de vacas por hato es Chimborazo. La media del número de vacas por hato en Chimborazo corresponde a 6, mayor que el promedio de Tungurahua y Cotopaxi, ambas con un promedio de 4 .
Por otra parte, en la Tabla 8 los resultados comparativos establecen que la provincia que presenta mayores diferencias en la variable cantidad de hectáreas cultivadas de pasto es Cotopaxi. La media de Cotopaxi es de 0,39 hectáreas de pasto cultivadas, menor que en las provincias de Chimborazo $(0,74$ ha) y Tungurahua $(0,76 \mathrm{ha})$. 
Tabla 7. Resultados del análisis de correlación por número de vacas por hato.

Variable dependiente: HSD Tukey

\section{Comparaciones múltiples}

\begin{tabular}{ccccc}
\hline \multirow{2}{*}{ (I) Provincias } & & $\begin{array}{c}\text { Diferencia } \\
\text { de medias (I-J) }\end{array}$ & $\begin{array}{c}\text { Error } \\
\text { estándar }\end{array}$ & Sig. \\
\hline \multirow{2}{*}{ TUNGURAHUA } & COTOPAXI &, $665^{*}$ & 0.232 & 0.012 \\
\cline { 2 - 5 } & CHIMBORAZO & $-1,869^{*}$ & 0.339 & 0 \\
\hline \multirow{2}{*}{ COTOPAXI } & TUNGURAHUA &,$- 665^{*}$ & 0.232 & 0.012 \\
\cline { 2 - 5 } & CHIMBORAZO & $-2,534^{*}$ & 0.334 & 0 \\
\hline \multirow{2}{*}{ CHIMBORAZO } & TUNGURAHUA & $1,869^{*}$ & 0.339 & 0 \\
\cline { 2 - 5 } & COTOPAXI & $2,534^{*}$ & 0.334 & 0 \\
\hline
\end{tabular}

*. La diferencia de medias es significativa en el nivel 0,05.

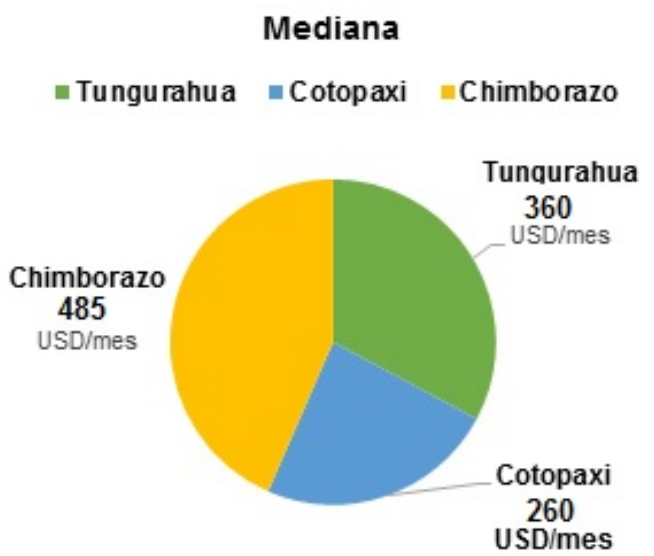

Figura 6. Comparativo de ingreso familiar por producción lechera por provincia.

En la Tabla 9 la provincia de Tungurahua exhibe mayores diferencias en la variable hectáreas naturales de pasto. La provincia de Tungurahua posee la mayor extensión de pastos naturales con una media de 1,10 ha, respecto de Cotopaxi $(0,65)$ y Chimborazo (0,45 ha).

En la Tabla 10 se observa que la provincia de Cotopaxi refleja las mayores diferencias respecto del ganado de raza Holstein con un promedio de 3 vacas (Holstein) por hato, inferiores a Tungurahua (5) y Chimborazo (4). Finalmente, la provincia de Chimborazo posee las mayores diferencias en asistencia técnica. El 65\% de los encuestados en Chimborazo dice no haber recibido asistencia técnica, es decir, que tan solo el $35 \%$ si lo ha hecho. Por otra parte, un $68 \%$ en Cotopaxi y un $69 \%$ en Tungurahua, indican que si recibieron asistencia técnica (Tabla 11).

\subsection{Discusión de los resultados}

Los resultados obtenidos en esta investigación demuestran que existen parámetros que incrementan la capacidad de los pequeños productores lecheros. Considerando el inequitativo acceso a factores de producción, en especial el tamaño de las explotaciones (1 ->10 ha) este resulta un limitante por la disponibilidad de pastos para los alimentos de las UBAs. En este sentido, se observa una concentración del número de vacas en el rango de entre 1-5 
Tabla 8. Resultados del análisis de correlación por cantidad de hectáreas de pasto cultivadas.

Variable dependiente: HSD Tukey

\section{Comparaciones múltiples}

\begin{tabular}{|c|c|c|c|c|}
\hline \multicolumn{2}{|c|}{ (I) Provincias } & \multirow{2}{*}{$\begin{array}{c}\begin{array}{c}\text { Diferencia } \\
\text { de medias (I-J) }\end{array} \\
, 37435^{*}\end{array}$} & \multirow{2}{*}{\begin{tabular}{|c|c|}
$\begin{array}{c}\text { Error } \\
\text { estándar }\end{array}$ \\
0.07029 \\
\end{tabular}} & \multirow{2}{*}{$\begin{array}{c}\text { Sig. } \\
0\end{array}$} \\
\hline TUNGUR AHUA & COTOPAXI & & & \\
\hline IUN UNATUA & CHIMBORAZO & 0.02267 & 0.08032 & 0.957 \\
\hline \multirow{2}{*}{ COTOPAXI } & TUNGURAHUA &,$- 37435^{*}$ & 0.07029 & 0 \\
\hline & CHIMBORAZO &,$- 35168^{*}$ & 0.05915 & 0 \\
\hline \multirow{2}{*}{ CHIMBORAZO } & TUNGURAHUA & -0.02267 & 0.08032 & 0.957 \\
\hline & COTOPAXI & ,35168* & 0.05915 & 0 \\
\hline
\end{tabular}

*. La diferencia de medias es significativa en el nivel 0,05.

Tabla 9. Resultados del análisis de correlación por cantidad de hectáreas de pastos naturales.

Comparaciones múltiples

Variable dependiente: HSD Tukey

\begin{tabular}{|c|c|c|c|c|}
\hline \multicolumn{2}{|c|}{ (I) Provincias } & \multirow{2}{*}{$\begin{array}{c}\begin{array}{c}\text { Diferencia } \\
\text { de medias (I-J) }\end{array} \\
, 44687^{*}\end{array}$} & \multirow{2}{*}{$\begin{array}{c}\begin{array}{c}\text { Error } \\
\text { estándar }\end{array} \\
0.07513\end{array}$} & \multirow{2}{*}{$\begin{array}{c}\text { Sig. } \\
0\end{array}$} \\
\hline & COTOPAXI & & & \\
\hline TUNGURAHUA & CHIMBORAZO &, $64429 *$ & 0.12157 & 0 \\
\hline \multirow{2}{*}{ COTOPAXI } & TUNGURAHUA &,$- 44687 *$ & 0.07513 & 0 \\
\hline & CHIMBORAZO & 0.19742 & 0.13105 & 0.289 \\
\hline \multirow{2}{*}{ CHIMBORAZO } & TUNGURAHUA &,$- 64429 *$ & 0.12157 & 0 \\
\hline & COTOPAXI & -0.19742 & 0.13105 & 0.289 \\
\hline
\end{tabular}

*. La diferencia de medias es significativa en el nivel 0,05 .

Tabla 10. Resultados del análisis de correlación en las tres provincias por cantidad de ganado de raza Holstein.

Variable dependiente: HSD Tukey

\section{Comparaciones múltiples}

\begin{tabular}{clcccc}
\hline \multirow{2}{*}{ (I) Provincias } & & $\begin{array}{c}\text { Diferencia } \\
\text { de medias (I-J) }\end{array}$ & $\begin{array}{c}\text { Error } \\
\text { estándar }\end{array}$ & Sig. \\
\hline \multirow{2}{*}{ TUNGURAHUA } & COTOPAXI & $1,439^{*}$ & 0.394 & 0.001 \\
\cline { 2 - 5 } & CHIMBORAZO & -0.739 & 0.468 & 0.256 \\
\hline \multirow{2}{*}{ COTOPAXI } & TUNGURAHUA & $-1,439^{*}$ & 0.394 & 0.001 \\
\cline { 2 - 5 } & CHIMBORAZO & $-2,178^{*}$ & 0.537 & 0 \\
\hline \multirow{2}{*}{ CHIMBORAZO } & TUNGURAHUA & 0.739 & 0.468 & 0.256 \\
\cline { 2 - 5 } & COTOPAXI & $2,178^{*}$ & 0.537 & 0 \\
\hline
\end{tabular}

*. La diferencia de medias es significativa en el nivel 0,05. 
Tabla 11. Resultados del análisis de correlación para las tres provincias por acceso a asistencia técnica

\begin{tabular}{ccccc}
\multicolumn{2}{c}{ Comparaciones múltiples } \\
Variable dependiente: HSD Tukey & & $\begin{array}{c}\text { Diferencia } \\
\text { de medias (I-J) }\end{array}$ & $\begin{array}{c}\text { Error } \\
\text { estándar }\end{array}$ & \multirow{2}{*}{ Sig. } \\
\hline \multirow{2}{*}{ (I) Provincias } & & -0.016 & 0.036 & 0.894 \\
\hline \multirow{2}{*}{ TUNGURAHUA } & COTOPAXI &,$- 346^{*}$ & 0.052 & 0 \\
\cline { 2 - 5 } & CHIMBORAZO & 0.016 & 0.036 & 0.894 \\
\hline \multirow{2}{*}{ COTOPAXI } & TUNGURAHUA &,$- 330^{*}$ & 0.051 & 0 \\
\cline { 2 - 5 } & CHIMBORAZO &, $346^{*}$ & 0.052 & 0 \\
\hline \multirow{2}{*}{ CHIMBORAZO } & TUNGURAHUA &, $330^{*}$ & 0.051 & 0 \\
\cline { 2 - 5 } & COTOPAXI & &
\end{tabular}

*. La diferencia de medias es significativa en el nivel 0,05.

UBAs en las tres provincias, destacándose por tener el mayor valor $(80,87 \%)$ la provincia de Cotopaxi. Además, en promedio la cantidad de leche producida por día en el hato es mayor para el rango entre 5-10 UBAs. Además, predomina el empleo de mano de obra familiar.

Las diferencias observadas a nivel de las tres provincias identifican que la provincia de Tungurahua mantiene un nivel más alto de participación de (a) asistencia técnica y (b) crédito para los productores, mientras que Cotopaxi se encuentra de segundo y Chimborazo con la menor participación. Al respecto, el tamaño de explotaciones por provincia varía en cuanto a su extensión. Es decir, en la provincia de Chimborazo existe una concentración entre 0,5-1 ha, Cotopaxi varía entre 0,1 y 1 ha, sin embargo, en Tungurahua esta concentración se amplía en el rango de 0,5 y 3 ha. Al respecto, se observa de forma general que las extensiones de alrededor de 1 ha pueden alcanzar ingresos de entre 250 y 500 USD/explotación. Sin embargo, las provincias de Cotopaxi y Tungurahua son aquellas que se encuentran en el umbral más bajo de los ingresos percibidos.

Por otra parte, las variables que presentan una correlación significativa son: tamaño del hato, tipo de ordeño, modernización de las instalaciones, raza de ganado y tipo de alimentación. En especial, esta condición se mantiene para las tres provincias. Es decir, que las políticas encaminadas a mejorar las capacidades de las explotaciones de agricultura familiar que incentiven estos factores pueden lograr incrementar los ingresos de los agricultores, así como la calidad de vida de las familias campesinas.

\section{Conclusiones}

La investigación tuvo como propósito analizar las dinámicas de los pequeños productores de leche de tres provincias que componen la Sierra centro de Ecuador: Cotopaxi, Tungurahua y Chimborazo, mediante un estudio que comprende 793 encuestas realizadas a pequeños productores. Los resultados alcanzados en este estudio describen las condiciones de la agricultura familiar, en específico la producción de leche. La tipología identificada a partir de la metodología permite reconocer a extensiones entre 1 y más de 10 hectáreas, como las explotaciones que predominan en la muestra analizada. En este sentido, se observan diferencias en las capacidades de las explotaciones por provincia. Es decir, en el caso de Tungurahua, existen mejores condiciones para desarrollar una producción lechera a pequeña escala, considerando las limitaciones por acceso a tierra, tecnificación y factores de producción.

El acceso de recursos que se relacionan con la modernización e innovación de los hatos representa un factor importante que incide positivamente en la producción y generación de ingresos familiares. Por lo que aquellas explotaciones en donde se ha incorporado la modernización de sus explotaciones (tecnología, alimentación y raza del ganado) favorecen a mejorar su productividad.

Finalmente, se concluye que la política pública a lo largo de los últimos años, en donde se introdujeron recursos para el desarrollo de la agricultura, no presenta una evidencia de mejora de las características de acceso a recursos para los pequeños 
productores, repercutiendo en la cantidad de ingresos familiares percibidos. Es decir, los niveles de ingresos se mantienen por debajo de la remuneración básica a nivel familiar, a pesar de que existen intentos porque el rendimiento supere la media nacional. Además, persisten las limitaciones de subparcelación y constitución de "microhatos" ganaderos que proveen de ingresos a las familias de productores, con limitaciones que lo perfilan como explotaciones de subsistencia.

\section{Referencias}

Barragán, Fernando (2010). «Redes espaciales de abastecimiento de lácteos en Ecuador». En: PRODIG-Universidad París 1 Panteón-Sorbona, Online: https:/ / bit.ly/2Z7Orxk.

Barsky, Osvaldo (1984). Acumulación campesina en el Ecuador. Vol. 1. Facultad Latinoamericana de Ciencias Sociales.

Bretón, Víctor (2012). Toacazo: en los Andes equinocciales tras la reforma agraria. 333.31091724. Online: https: / / bit.ly / 2Z5BQ9G: FLACSO-ABYA YALA.

Chiriboga, M. (2003). «Innovación, Conocimiento y Desarrollo Rural». En: Segundo Encuentro de la Innovación y el Conocimiento para Eliminar la Pobreza Rural, Online: https://bit.ly/303DdHv.

Corporación Financiera Nacional (2017). FICHA SECTORIAL: Cría y Reproducción de Ganado Bovino. Inf. téc. Online: https: / / bit.ly /304RqUm: Corporación Financiera Nacional.

De Janvry, Alain y Elisabeth Sadoulet (2006). «Progress in the modeling of rural households' behavior under market failures». En: Poverty, inequality and development. Springer, 155-181. Online: https://bit.ly/2OVn3ig.

Escobar, Germán y Julio Berdegué (1990). «Tipificación de sistemas de producción agrícola». En: Online: https://bit.ly/2uD9tmH.

FAO (2013). FAO: la agricultura familiar produce más del $70 \%$ de los alimentos de Centroamérica. Inf. téc. Online: https://bit.ly/2KPjswH.

Franco-Crespo, Christian y Jose Sumpsi Viñas (2017). «The Impact of Pricing Policies on Irrigation Water for Agro-Food Farms in Ecuador». En: Sustainability 9.9, 1515. Online: https: / / bit. ly/33B7HCX.
Glikman, P. (1991). Encadenamientos de producción en la economía campesina en el Ecuador. Vol. 1. Online: https://bit.ly/2L5SO4n: Agroamerica.

Grisa, Catia y Sergio Schneider (2008). «"Plantar pro gasto": a importância do autoconsumo entre famílias de agricultores do Rio Grande do Sul». En: Revista de Economia e Sociologia Rural 46.2, 481-515. Online: https:/ / bit.ly/2ZZxFh4.

ILDES (1988). El problema agrario en el Ecuador. ILDIS.

INEC, ed. (2016). Encuesta de Superficie y Produccion Agropecuaria Continua. INEC.

Larrea, Carlos y col. (2008). «Mapas de pobreza, consumo por habitante y desigualdad social en el Ecuador: 1995-2006; metodología y resultados». En: Documento de trabajo/Programa Dinámicas Territoriales Rurales. RIMISP-Centro Latinoamericano para el Desarrollo Rural; no. 13, Online: https:/ / bit.ly/33yevRI.

Louhichi, Kamel y Sergio Paloma (2014). «A farm household model for agri-food policy analysis in developing countries: Application to smallholder farmers in Sierra Leone». En: Food Policy 45, 1-13. Online: https:/ / bit.ly/2MimkW7.

MAGAP (2016). La política agropecuaria ecuatoriana. Inf. téc. Ministerio de Agricultura, Ganadería, Acuacultura y Pesca.

Mantilla, F. (2015). «Técnicas de muestreo. Un enfoque en la investigación de Mercados.» En: Universidad de las Fuerzas Armadas-ESPE, Online: https://bit.ly/2NFuCrd.

Mena-Vásconez, Patricio, Rutgerd Boelens y Jeroen Vos (2016). «Food or flowers? Contested transformations of community food security and water use priorities under new legal and market regimes in Ecuador's highlands». En: Journal of Rural Studies 44, 227-238. Online: https:/ / bit.ly / 302pYGN.

Mumba, C. y col. (2011). «Economic analysis of the viability of smallholder dairy farming in Zambia». En: Foot 42, 26-8. Online: https: / / bit.ly / 2KQaAHp.

Murphy, M. y col. (2014). "Comparison of modelling techniques for milk-production forecasting». En: Journal of dairy science 97.6, 3352-3363. Online: https:/ / bit.ly/2Z7YgqW.

OECD-FAO (2015). Agricultural Outlook 2015-2044. Online OECD.

Ormel, P. (2013). América Latina y el Caribe en el contexto mundial de los productos de origen animal: Leche vacuna. 
Requelme, N. y N. Bonifaz (2012). «Carcaterización de sistemas de producción lechera de Ecuador». En: La Granja 27.1, 68-109. Online: https: / / bit. ly/2TFJfOJ.

Schejtman, A. (2008). La Agricultura Familiar en América Latina.

Steinfeld, H. y P. Chilonda (2006). Perspectiva Mundial. Roma.

Sumpsi, J. M. (2010). El nuevo sistema agroalimentario en una crisis global. Madrid.
Tobar, Jaime (2010). Criterios de tipificación y caracterización de la Agricultura Familiar en El Salvador. Inf. téc. Online: https: / / bit.ly / 2InFEQv, 1-12. Online: https:/ / bit.ly/2InFEQv.

Wiggins, Steve, Johann Kirsten y Luis Llambí (2010). «The future of small farms». En: World development 38.10, 1341-1348. Online: https: / / bit. ly/2TvLVvC. 